\title{
El reglamento de actividades arqueológicas y la investigación paleontológica en Andalucía: ¿El estudio del registro fósil debe ser competencia de los licenciados en humanidades?
}

\author{
Paul Palmqvist Barrena | Dpto. de Ecología y Geología, Universidad de Málaga \\ Matías Reolid | Dpto. de Geología, Universidad de Jaén \\ Borja Figueirido, Juan Antonio Pérez-Claros, Antonio Guerra-Merchán, José Manuel García-Aguilar, Francisco J. Serrano \\ | Dpto. de Ecología y Geología, Universidad de Málaga
}

URL de la contribución <www.iaph.es/revistaph/index.php/revistaph/article/view/4164>

España atesora un patrimonio cultural ingente y diverso, de carácter tanto histórico como natural, y su registro fósil no es en absoluto una excepción. Así, una serie de enclaves de nuestra geografía gozan de reconocimiento a nivel mundial gracias a su extraordinario patrimonio paleontológico, e igualmente debido a las investigaciones que diversos equipos científicos vienen realizando sobre el mismo. Es el caso de los yacimientos pleistocenos de la sierra burgalesa de Atapuerca (Burgos), en los que se continúa reescribiendo la evolución humana, del humedal cretácico de las Hoyas (Cuenca), donde aprendemos día a día sobre la evolución de las aves y los mamíferos mesozoicos, o los afloramientos cámbricos de Murero (Zaragoza), que aportan información clave sobre la historia temprana de los metazoos a comienzos del Paleozoico, por solo citar tres emplazamientos particularmente destacados.

Andalucía alberga, igualmente, un patrimonio fósil de proyección internacional, como ocurre con los importantes yacimientos paleontológicos de la región de Orce en el altiplano granadino (depresión de Guadix-Baza), los cuales atesoran las evidencias más antiguas de presencia humana en el margen occidental de Europa (1,4 millones de años), lo que nos abre una ventana excepcional al estudio de los ecosistemas peninsulares de inicios del Cuaternario, o los yacimientos marinos de la comarca almeriense de Cuevas del Almanzora, por el momento menos estudiados, que nos transportan a un mundo de manglares subtropicales habitado por cetá- ceos y manatíes hace más de cuatro millones de años. Por ello, nuestra administración debería tener como prioridad la investigación del patrimonio paleontológico que atesoran tales enclaves y muchos otros, investigación que se debe orientar hacia la diseminación del conocimiento científico y la integración social de dichos recursos como bienes de carácter cultural, vertebradores del desarrollo económico del territorio en el que se ubican.

Por otra parte, no podemos olvidar que, conforme a la recomendación de la UNESCO, la idea de patrimonio se debe desarrollar desde una perspectiva más amplia, basada en el concepto de patrimonio de naturaleza inmaterial, lo que es de aplicación también en el ámbito de la Paleontología. Este enfoque, concebido inicialmente para los hechos culturales, puede incluir igualmente a los científicos o a cualesquiera otros que deriven de los logros de la humanidad, lo que nos lleva desde la idea más o menos inmediata de que los fósiles son objetos patrimoniales singulares, que deben conservarse en los museos, a la noción de que son los conocimientos paleontológicos generados tras su estudio los que verdaderamente dan valor a ese patrimonio. Así, las explicaciones llegan con frecuencia a ser más interesantes que la belleza o el interés que pueda despertar en sí mismo un fósil. Los yacimientos de la región de Orce vuelven a ser un buen ejemplo de tales prácticas en Andalucía, que contribuyen a la puesta en valor del patrimonio histórico y natural. Durante las últimas décadas, la investigación del contexto geológico, los atributos tafonómicos 
y el marco paleoecológico de las asociaciones fósiles de grandes mamíferos recuperadas en los yacimientos orcenses, en particular Venta Micena, Barranco León-D y Fuente Nueva-3, ha permitido constatar la dispersión, hace un millón y medio de años, de fauna africana hacia la ribera norte del Mediterráneo, entre cuyos integrantes se encuentran los primeros pobladores humanos de Eurasia, conservándose tales vestigios en las inmediaciones de los sistemas lacustres de la cuenca de Baza, que estaban sujetos a surgencias de aguas termales. En estos ecosistemas tan singulares, los fósiles de la fauna de grandes mamíferos que habitaban en las inmediaciones de los lagos se conservaron en el entorno de los cubiles de cría de las hienas gigantes (Pachycrocuta brevirostris) que poblaban la cuenca, como ocurre en el caso de Venta Micena. Tales hienas competían intensamente con los homininos por el acceso a la carroña, según se evidencia particularmente en Fuente Nueva-

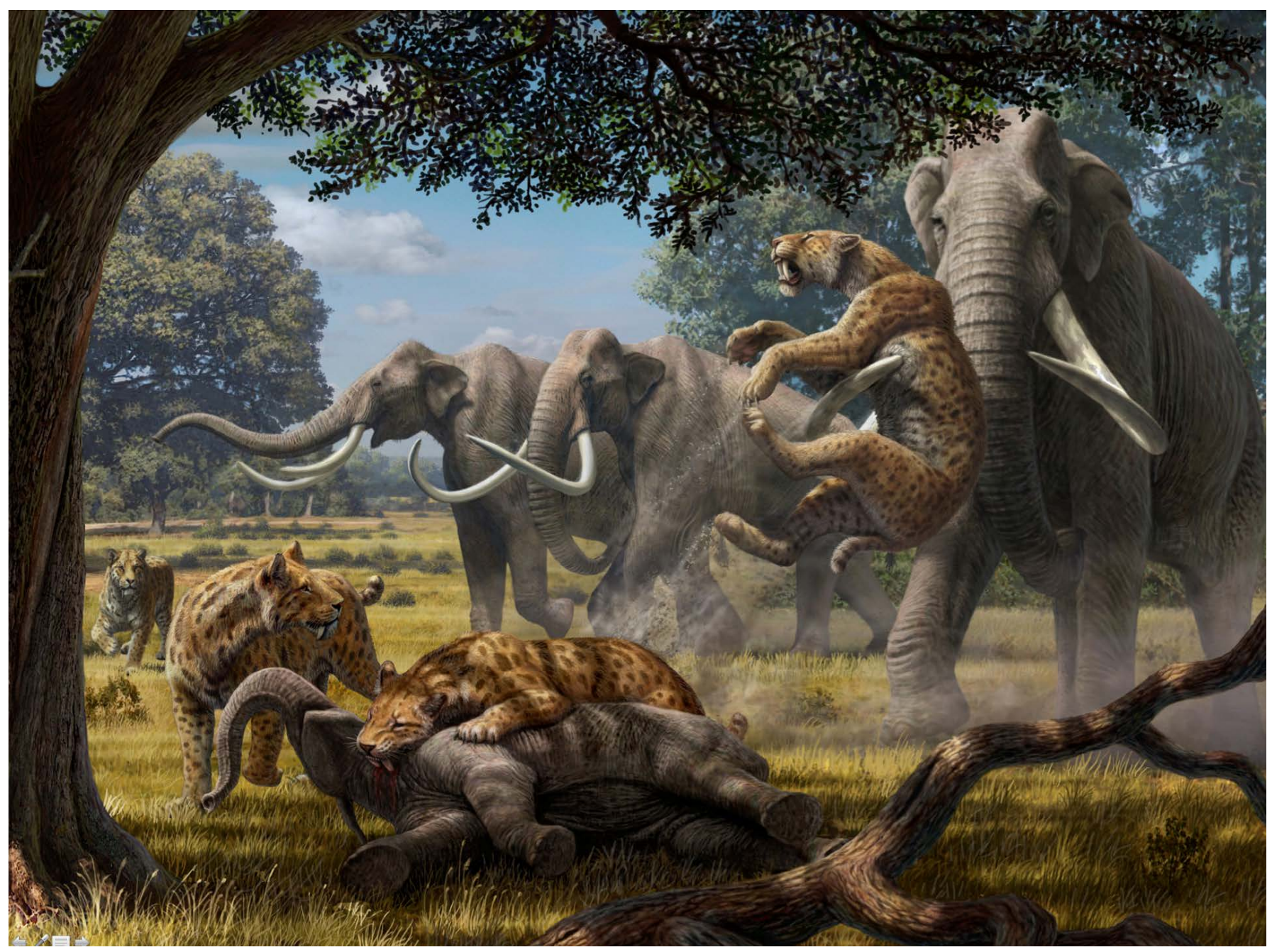

Reconstrucción del entorno de Orce hace 1,4 millones de años. Se aprecia, en primer plano, a tres félidos con dientes de sable de la especie Homotherium latidens abatiendo a una cría del elefante Mammuthus meridionalis, mientras en segundo término intervienen defensivamente tres ejemplares adultos para separar a uno de ellos de su presa y ahuyentar a un cuarto depredador | dibujo Mauricio Antón 
a debate El marco legal para la protección del patrimonio paleontológico. ¿Qué pasa en tu comunidad?

3 , un verdadero cementerio de elefantes, dejando como testimonio ingentes asociaciones de útiles líticos tallados in situ según la tradición olduvayense y, en el caso de Barranco León-D, un diente de leche que es hoy por hoy el fósil humano más antiguo de Europa.

Desgraciadamente, la labor investigadora de los paleontólogos no se ve facilitada en nuestra comunidad por la administración, más bien al contrario. Así, el Reglamento de Actividades Arqueológicas de Andalucía (BOJA núm. 134, DECRETO 168/2003 de 17 de junio), que regula la normativa aplicable a la prospección y excavación de este patrimonio, define en su artículo 2 (apartado a) como excavación arqueológica la destinada al descubrimiento de restos históricos o paleontológicos, e incluso los componentes geomorfológicos relacionados con ellos, consideración que se hace extensiva a la prospección arqueológica (apartado b), entendida como exploración dirigida al estudio, investigación o detección de vestigios arqueológicos o paleontológicos.

Por otra parte, el artículo 3, relativo a los tipos de excavaciones arqueológicas, contempla como tales las excavaciones extensivas en superficie para documentar el registro estratigráfico y extraer científicamente los vestigios paleontológicos, e igualmente considera como sondeos arqueológicos los análisis de las secuencias estratigráficas de los yacimientos, poniendo incluso en manos de los arqueólogos el control de los movimientos de tierras, en detrimento de los especialistas en geotecnia.

Finalmente, en el artículo 6 se explicita quiénes pueden solicitar autorización a la administración andaluza para realizar este tipo de actividades, consideradas como exclusivamente arqueológicas, indicando que son únicamente los licenciados en humanidades con formación teórico-práctica acreditada en arqueología o los departamentos universitarios y museos competentes en arqueología.

Por todo ello, y aunque a priori pueda resultar difícil de creer, el reglamento excluye categóricamente de la dirección de tales estudios y excavaciones a los propios paleontólogos, incluso en el caso de aquellos yacimientos que son de naturaleza exclusivamente paleontológica (esto es, sin evidencias culturales de la humanidad, como ocurre en la mayoría de los casos, pues el registro fósil comprende más de 3.500 millones de años), siendo que nuestra comunidad cuenta con algunos de los equipos paleontológicos de trayectoria científica más acreditada en su especialidad a nivel internacional.

Así pues, esta reglamentación anula claramente las competencias profesionales de los paleontólogos y, en general, de los geólogos en Andalucía, lesionando sus legítimos intereses profesionales al supeditar la dirección de una serie de actividades científicas, como las prospecciones geológicas y las excavaciones paleontológicas, a los licenciados en humanidades. Esto lleva a pensar que dicho reglamento de actividades arqueológicas, en su versión del año 2003, actualmente vigente, se elaboró con la

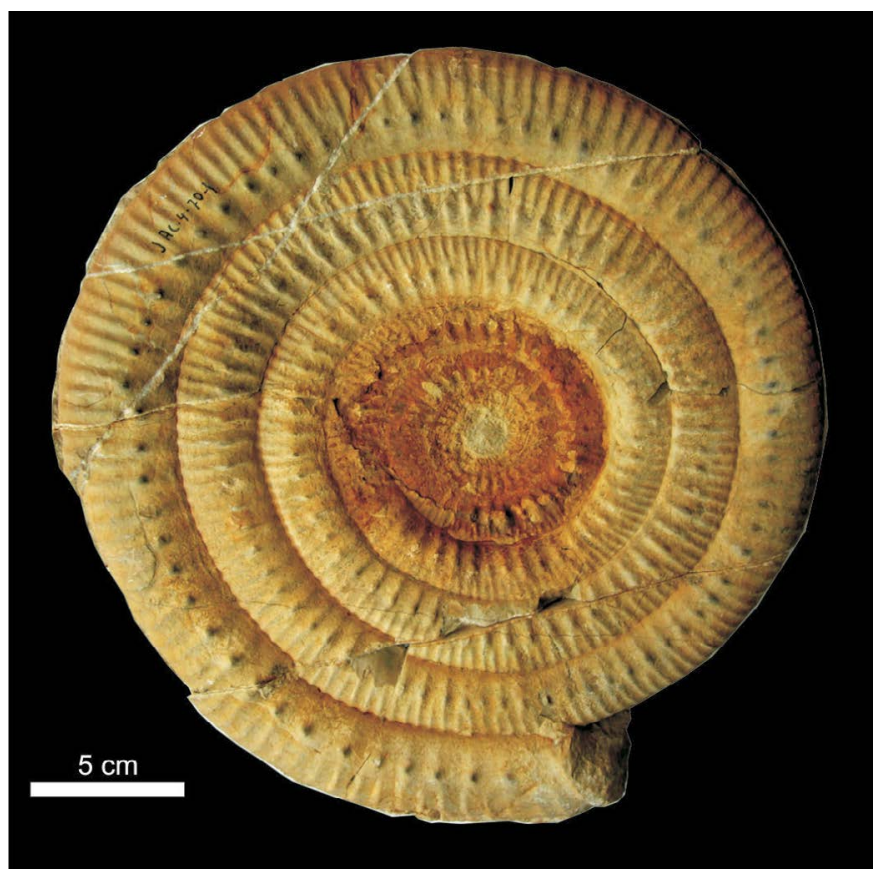

Ammonites del género Skirroceras del Bajociense (169 millones de años, Jurásico medio) de Campillo de Arenas (provincia de Jaén, Subbético Medio) | foto Matías Reolid 
_a debate El marco legal para la protección del patrimonio paleontológico. ¿Qué pasa en tu comunidad?

intención de dotar de la máxima inclusividad posible a las actividades profesionales de los arqueólogos y los prehistoriadores, dejando a su cargo competencias científicas que en buena lógica deberían haber recaído en otros profesionales, como los paleontólogos y los estratígrafos. Por ello, cabe preguntarse entonces hasta qué punto incurre el reglamento en una clara prevaricación administrativa. De hecho, en la versión anterior del reglamento, de 1993, se manifestaba que podrán solicitar autorización para realizar actividades arqueológicas las personas físicas, nacionales o extranjeras, que cuenten con la titulación académica de Licenciado, y acrediten formación arqueológica o paleontológica. Esto induce a pensar que en la versión posterior del mismo se excluyó deliberadamente a los paleontólogos. Además, en el reglamento de 1993 se indicaba, en su artículo 3, que para las excavaciones sistemáticas terrestres y las prospecciones con sondeos estratigráficos terrestres, será necesaria la presencia en el equipo de un arquitecto o arquitecto técnico, lo cual se eliminó también en la versión vigente de 2003, donde tales competencias profesionales, claramente ajenas a la formación de los arqueólogos, recaen una vez más en los licenciados en humanidades.

Por otra parte, en el reglamento de 2003 no se habla en ningún momento de restringir las actividades consideradas como propiamente arqueológicas al Cuaternario (últimos 2,6 millones de años), período en el que cabe contemplar la presencia simultánea en los yacimientos de restos humanos, que son competencia de los paleoantropólogos, y sus manifestaciones culturales, como las industrias líticas, que son competencia de los arqueólogos (aunque como icnofósiles, esto es, evidencias de la actividad vital de una especie extinta, también interesan a los paleontólogos, tal y como ocurre con las huellas de los dinosaurios).

De hecho, en el caso de los yacimientos de Orce, como sucede en Fuente Nueva-3 y Barranco León-D, se presentan ambos tipos de registros, industrias líticas (que representan menos del $15 \%$ de los registros) y fósiles de macrovertebrados (la gran mayoría de los restos, a los que se sumarían los fósiles de la microfauna). No obstante, la dirección de las excavaciones en estos yacimientos ha recaído durante las dos últimas décadas siempre en manos de arqueólogos, pese a su falta de competencias profesionales para trabajar con restos paleontológicos.

Más aún, esta situación afecta también al yacimiento de Venta Micena, que es exclusivamente paleontológico, pues su cronología es anterior a la llegada de las primeras poblaciones humanas a nuestras latitudes, pese a lo cual su excavación continúa siendo dirigida por licenciados o graduados en humanidades. De hecho, en el reglamento de actividades arqueológicas no se explicita que su ámbito de aplicación se restrinja a aquellos yacimientos en los que se constate la presencia humana o las evidencias de actividad antrópica. Por ello, si sumamos esta consideración a la efectuada anteriormente, en el sentido de que su regulación no se limita a los yacimientos pleistocenos, en los que sí cabe contemplar la posibilidad de dicha presencia humana, debería entenderse, por extensión, que este reglamento afecta potencialmente a cualquier resto fósil o yacimiento paleontológico (por ejemplo, los trilobites del Cámbrico o los dinosaurios del Mesozoico), lo cual podría generar en el futuro un problema de competencias aún mayor que el planteado actualmente.

Esta carencia de habilidades profesionales para gestionar la investigación del registro fósil tiene su expresión más perniciosa en el riesgo potencial de destrucción del patrimonio paleontológico durante la propia excavación, al carecer el arqueólogo de la formación necesaria, tanto de índole geológica como biológica, para afrontar la extracción de los fósiles. Así, cabe plantearse qué conocimientos puede tener un licenciado en humanidades sobre la anatomía de los ammonites jurásicos o la de los notosaurios triásicos, sobre los paleoecosistemas en los que vivieron estos organismos (los cuales carecen de representantes actuales) o sobre las características de las propias rocas en las que se conservaron sus fósiles.

Este tema ha sido objeto de numerosos debates en las jornadas que celebra anualmente la Sociedad Española 
a debate El marco legal para la protección del patrimonio paleontológico. ¿Qué pasa en tu comunidad?

de Paleontología, particularmente en el seno de su Grupo de Trabajo sobre Patrimonio Paleontológico. En todo caso, pese a tales agravios y limitaciones, conviene recordar que han sido paleontólogos y geólogos los que han generado el grueso del conocimiento que se tiene sobre el extraordinario patrimonio fósil andaluz. Así, en el caso de los importantes yacimientos de la depresión de Guadix-Baza, que se llevan excavando y estudiando durante décadas, la inmensa mayoría de las publicaciones con índice de calidad y repercusión internacional han sido realizadas por equipos de investigación coordinados por paleontólogos, lo que ha generado un considerable impacto y difusión en los medios de comunicación, contribuyendo a la puesta en valor y socialización de este extraordinario patrimonio.

A modo de resumen, y en función de todo lo expuesto anteriormente, consideramos que el reglamento de actividades arqueológicas de Andalucía, en su versión actual, lesiona gravemente las competencias profesionales de los paleontólogos y, por extensión, de toda una serie de especialistas del ámbito de la Biología y la Geología, como los estratígrafos, los geocronólogos, los geomorfólogos, los paleobiólogos, los palinólogos, los sedimentólogos y los tafónomos, al situar la responsabilidad del estudio y la gestión de los bienes paleontológicos en manos de licenciados en humanidades con experiencia válida en Arqueología. Tal reglamentación, única en España, sienta graves precedentes y limita seriamente las investigaciones paleontológicas que se desarrollan sobre el ingente registro fósil que atesora nuestra comunidad autónoma.

Por otra parte, la ausencia de reconocimiento por parte de la administración de las competencias profesionales de los paleontólogos genera un vacío legal en lo relativo a la protección de los propios yacimientos paleontológicos, que en la mayoría de los casos no cuentan con las mínimas medidas de protección exigibles. Es el caso, entre otros, del yacimiento cárstico de Moreda en Guadix-Baza, generado por la actividad de las aves rapaces, el cual atesora incontables restos de micromamíferos, como insectívoros, roedores y murciélagos de la época pliocena, así como el primer registro conocido en nuestra comunidad del primate Paradolicopithecus arvernensis, un macaco de anatomía convergente con la de los geladas modernos. Este enclave, apenas estudiado, está siendo sometido a un expolio continuado por parte de los coleccionistas, encontrándose fósiles del mismo a la venta en mercadillos y en la web, lo que traslada una imagen penosa de la ausencia de interés por parte de nuestra administración en la salvaguarda de tales recursos patrimoniales, los cuales no representan ninguna prioridad para los arqueólogos a los que se les han concedido las competencias sobre su estudio. 